Sociologie et sociétés

\title{
" Je me souviens "... en chansons : articulations de la citoyenneté culturelle et de l'identitaire dans le champ musical au Québec
}

\author{
"Je me souviens"... in Song: Articulations of Cultural \\ Citizenship and Identitty in the Field of Popular Music in \\ Quebec
}

\section{Line GRENIER}

Volume 29, numéro 2, automne 1997

La mémoire sociale

URI : https://id.erudit.org/iderudit/001312ar

DOI : https://doi.org/10.7202/001312ar

Aller au sommaire du numéro

Éditeur(s)

Les Presses de l'Université de Montréal

ISSN

0038-030X (imprimé)

1492-1375 (numérique)

Découvrir la revue

Citer cet article

GRENIER, L. (1997). « Je me souviens "... en chansons : articulations de la citoyenneté culturelle et de l'identitaire dans le champ musical au Québec. Sociologie et sociétés, 29(2), 31-47. https://doi.org/10.7202/001312ar

\section{Résumé de l'article}

L'auteure s'intéresse à la première exposition d'envergure nationale consacrée à la musique populaire québécoise, " Je vous entends chanter ", en tant que terrain clé pour l'étude du dispositif qui rend possible l'articulation de ce domaine musical comme lieu privilégié de production de la citoyenneté culturelle et de l'identitaire au Québec. Les documents et artefacts composant l'exposition, ainsi que la couverture médiatique dont elle a fait l'objet, servent ici à l'examen des conditions matérielles, institutionnelles et discursives, qui concourent à faire de l'histoire de la musique populaire celle du " peuple québécois ". L'analyse explore le jeu complexe des mémoires qui médiatisent les façons contrastées dont cet événement muséal dit, nomme et raconte la place de la musique populaire québécoise et, ce faisant, les formes d'allégeance, d'identification et d'appartenance qui y sont articulées. 


\section{« Je me souviens »... en chansons : articulations de la citoyenneté culturelle et de l'identitaire dans le champ musical populaire au Québec}

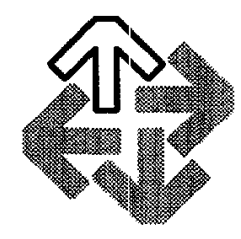

\section{L.INE GRENIER}

Le Musée de la civilisation de la ville de Québec a été l'instigateur et l'hôte de la première exposition d'envergure nationale consacrée à la musique populaire du Québec. Intitulée « Je vous entends chanter ", elle a été inaugurée quelques semaines avant la tenue du référendum de l'automne 1995 sur la souveraineté du Québec. Elle a pris fin en octobre 1996, après avoir remporté un succès sans précédent auprès du public comme de la critique ${ }^{1}$.

Inaginez-vous entrant dans une pièce à l'éclairage tamisé, muni d'un casque d'écoute à infrarouge. Des voix, majoritairement féminines, vous accueillent en entonnant un air folklorique familier, dans lequel vous avez d'ailleurs peut-être reconnu le thème musical de l'édition 1996 de la parade de la Saint-Jean-Baptiste, fête nationale du Québec : " $A$ la claire fontaine/ m'en allant promener/j'ai trouvé l'eau si belle/que je m'y suis baignée/Il y a longtemps que je t'aime/ jamais je ne t'oublierai. » Alors que ce chant se transforme en murmure sourd, une voix d'homme vous souhaite la bienvenue à l'exposition :

Je vais vous raconter une histoire d'amour, celle qui existe depuis 400 ans entre le Québec francophone et sa chanson. C'est l'histoire d'un peuple et d'une chanson improbables, perdus dans un univers anglophone et ballotté entre les grands courants venus d'ailleurs. Depuis toujours, la chanson a accompagné les Québécois. Elle a reflété fidèlement leurs aspirations, leurs rêves, leur identité. Après avoir écouté, imité et assimilé toutes les influences, la chanson d'ici s'est enfin assumée. (Musée de la civilisation - MC - 1996, F. 7.)

Considérant l'exposition «Je vous entends chanter » comme un moment clé du vaste discourss public dont la musique populaire est l'objet au Québec, le présent article propose un diagnostic des rapports de savoir/pouvoir (Foucault, 1980) qui ont présidé à l'intronisation de ladite chanson québécoise au temple de la muséologie nationale contemporaine au Québec. Je soulaaite mettre en évidence les conditions qui concourent à poser le domaine musical dit

1. Depuis mars 1997 , l'exposition a rouvert ses portes dans les locaux d'un musée montréalais, le Musée Juste pour Rire, où elle continue à attirer les foules. Il n'en sera malheureusement pas question dans cet article. Car bien qu'aucune transformation majeure n'ait été apportée à l'exposition proprement dite, le lieu qui l'abrite, le contexte institutionnel dans lequel elle s'inscrit, la composition distincte des publics cibles ainsi que la forte présence des agents musicaux et la place centrale qu'occupent les médias dans la métropole culturelle qu'est Montréal, notamment, en font un événement distinct, justiciable d'une nouvelle analyse. 
populaire en partenaire obligé de la nationalité comme de la citoyenneté et, dès lors, à faire inexorablement de l'histoire de cette musique celle du «peuple québécois ». Dans la mesure où cet événement se veut une invitation à se souvenir, je désire aussi examiner les formes de mémoires distinctes dont est tributaire la production de cette histoire, ainsi que les formes de leur appropriation à travers lesquelles sont institués les lieux d'où il est légitime de parler de musique québécoise, de même que les espaces sociaux qu'elle occupe.

\section{VERS L'ANALYSE D'UN DISPOSITIF}

L'analyse qui suit s'inscrit dans le cadre d'un programme de recherche consacré à l'étude des processus de «mise en société " (Hennion, 1993) de la musique populaire francophone au Québec, c'est-à-dire des rapports itératifs dans et par lesquels des objets (disques, spectacles, partitions, etc.) et des sujets (auditeurs, compositeurs, producteurs, etc.) sont réciproquement définis comme « musicaux » et déterminent, dès lors, ce qui vaut et peut valoir comme « musique populaire québécoise " à un moment donné. Sa visée est de mieux comprendre la manière dont sont établies les frontières de ce champ culturel, ainsi que les conditions qui permettent que ce domaine d'activité s'impose à titre d'emblème par excellence de la culture québécoise moderne en tant que culture populaire nationale. À mon avis, un tel statut n'est attribuable aux propriétés présumément intrinsèques, génériques ou ontologiques du musical ; il est plutôt tributaire du dispositif particulier à travers lequel la musique populaire est produite au Québec : un assemblage historiquement contingent de discours et d'institutions spécifiques qui, soutenant des systèmes de savoir et d'action musicaux tout en étant supportés par eux, permettent d'orienter, de guider ou d'affecter la conduite de soi et des autres (Foucault, 1994).

Je me suis intéressée à l'exposition " Je vous entends chanter ", voire aux documents et aux artefacts qui la composent, de même qu'aux textes médiatiques et aux déclarations officielles qui contribuent à l'inscrire dans le discours public sur la musique populaire, en tant que partie intégrante de ce dispositif - laissant à d'autres le soin d'aborder les questions proprement muséologiques qu'elle soulève. À travers ce système de savoir/pouvoir opère un " régime de vérité » (Foucault, 1994a) qui, comme l'analyse empirique le mettra en évidence, régularise la production d'énoncés construisant la musique populaire à la fois comme « l'affaire de tout le monde ", comme un phénomène politiquement important et comme le terrain d'une lutte unique qui unit la destinée d'une nation à celle de ses agents culturels, individuels et corporatifs. En mettant en cuvre un tel régime - qu'elle contribue à produire - l'exposition a pour effet de définir la «place» de la musique québécoise : non pas les frontières de l'aire géographique qu'elle occuperait, mais plutôt la constellation d'interactions particulières, de relations sociales, de processus sociaux, d'expériences et de compréhensions [understandings], en situation de coprésence (Massey, 1993, p. 68, traduction L. G.) qui, en la régulant, la constituent. Mon intervention critique vise à montrer comment la définition de cette place est informée par le jeu complexe des mémoires construites et convoquées par l'exposition, lesquelles permettent de lier différents ordres de matériaux culturels à ce que j'appelle, à l'instar d'Allor (1997), les passés " présents " d'expériences individuelles et de trajectoires collectives. Pour ce faire, je mettrai l'accent sur la manière dont la place de la musique québécoise est simultanément dite, nommée et racontée en ce lieu précis. En insistant à la fois sur les proximités et les disjonctions observables sur ces trois plans, l'analyse discursive que je propose vise aussi à mettre en évidence comment « Je vous entends chanter » produit une articulation ${ }^{2}$ singulière de l'identitaire et de la citoyenneté qui rend possibles des formes spécifiques d'allégeance et d'appartenance au Québec en tant que communauté culturelle imaginée (imagined community) (Anderson, 1991).

2. M'inspirant de Hall (1986), j'entends par articulation la forme d'un lien ou assemblage qui, bien qu'il ne soit pas nécessaire, absolu, prédéterminé ou essentiel, permet néanmoins, dans certaines conditions, de créer une unité à partir d'éléments différents. Pour plus de détails, voir (SLACK), 1996. 
« JE ME SOUVIENS \# ... EN CHANSONS : ARTICULATIONS DE LA CITOYENNETÉ CULTURELLE ET DE L'IDENTITAIRE DANS LE CHAMP MUSICAL POPULAIRE AU QUÉBEC

\section{LA MUSIQUE POPULAIRE ENTRE AU MUSÉE}

L'intronisation initiale de la musique populaire ne s'est pas effectuée dans un musée d'art mais dans un musée dit « de société » où, sous la gouverne des sciences humaines, sont mises en valeur diverses facettes d'« un monde en continuité et en devenir » (Le Ménestrel, 1996, p. 74). Il s'agit d'une institution nationale qui se définit comme un musée thématique voué d'abord à la culture, l'histoire et la civilisation québécoises ; il ne vise pas à consacrer quelque chef-d'œeuvre ou à préserver pour la postérité quelque trésor d'hier, mais bien à offrir au public visiteur des expositions qui témoignent « de la vie humaine et des rapports de l'homme avec son histoire quotidienne et avec l'histoire " (op. cit., p. 75). La nature du mandat du Musée de la civilisation, combinée à la place privilégiée qu'occupent la musique et plus particulièrement la chanson au sein de la culture populaire québécoise, rendait presque inévitable la tenue d'une exposition sur un thème qui, de l'avis unanime des membres des équipes de conception, de recherche et de production, permet d'offrir au grand public francophone du Québec un " miroir sonore» dans lequel il se reconnaît (Bilodeau, 1994).

« Je vous entends chanter » regroupe environ 550 objets, 655 chansons, 300 artistes et plus de 30 heures d'enregistrements audio et vidéo. La partie centrale de l'exposition se présente sous la forme d'un grand demi-cercle composé d'une série de zones, dont les limites sont définies tant visuellement qu'auditivement ${ }^{3}$, comportant chacune un panneau d'identification, des écrans vidéos, des photos d'artistes associés à une période ou à un domaine particulier de musique populaire au Québec, ainsi qu'une panoplie d'objets et d'effets personnels prêtés pour l'occasion par les artistes auxquels ils appartiennent - allant du coffre métallique ayant servi aux tournées du groupe rock Offenback à la robe de mariée de la superstar Céline Dion. En vous rapprochant d'une des composantes matérielles d'une zone donnée, vous activez l'une ou l'autre des trois trames sonores suivantes : une mise en contexte de la période ou du domaine visé par cette zone, présentée par un narrateur; des vidéoclips et des extraits filmés de concerts extérieurs ou de performances télévisuelles; ou des entrevues, la plupart originales, avec des artistes jugés représentatifs de cette zone, qui commentent certaines de leurs créations et quelques-uns des faits marquants de leur carrière. Si vous vous éloignez de ces composantes matérielles, vous entendez un passage d'un pot-pourri destiné à présenter certaines des chansons les plus populaires ou significatives de la zone dans laquelle vous circulez.

\section{LA MISE EN MUSÉE DE LA CHANSON QUÉBÉCOISE}

\section{* DIRE » : VOIX PUBLIQUE ET VOIX DE L'INTIME}

En accordant une place prépondérante à plusieurs centaines de pièces du répertoire québécois, «Je vous entends chanter » semble permettre à la musique populaire de se dire elle-même, sans intermédiaire. L'exposition est pourtant tributaire d'un ensemble de médiations qui sont déterminantes de la manière dont la musique québécoise s'y trouve discursivement produite. Aux textes chantés, fruits d'un travail névralgique de sélection et de classification, se greffent en effet des textes parlés produits expressément pour l'exposition. Je me suis arrêtée sur ces derniers puisqu'ils rendent davantage accessibles les médiations qui informent cette autre instance du dire, d'ailleurs non moins instrumentale que les chansons elles-mêmes dans la production de la musique populaire comme phénomène social à part entière.

Les bandes audio auxquelles donne accès le casque d'écoute proposent deux séries de textes inédits, soient les mises en contexte du narrateur et les commentaires d'artistes. Ces textes résultent de pratiques spécifiques d'énonciation dont les traces matérielles peuvent être vues comme des marqueurs de subjectivité dans le discours (Havercroft, 1993). Je ne renvoie

3. L'analyse ne tient pas compte de la partie «Les immortelles " qui est dédiée entièrement à l'écoute de l'une ou de l'autre des 450 pièces sélectionnées par les membres de l'équipe de l'exposition pour représenter les diverses tendances constitutives du vaste répertoire de la musique québécoise, certaines de ses chansons les plus populaires ou les plus significatives. 
pas ici à quelque subjectivité immanente dont ces textes proposeraient la synthèse unifiante mais plutôt, m'inspirant des travaux de Foucault, aux « réseaux d'emplacement distincts » que déploie le discours comme "espace d'extériorité » (Foucault, 1969, p. 74). En ce sens, les modalités énonciatives constituent une voie d'accès stratégique au « qui » du discours (qui est fondé à tenir tel ou tel langage ?), à ses lieux institutionnels (d'où est-il possible de tenir ce langage ?) et aux positions de sujets qu'il produit (quelle relation entretient ce «qui » avec les objets et les informations que mobilise le langage tenu ?) (op. cit., p. 68-71).

Vues sous l'angle de l'énonciation, les mises en contexte sont caractérisées par un effacement quasi-complet du narrateur qui concourt, me semble-t-il, à produire un effet de détachement, sinon d'objectivité. Le narrateur ne marque explicitement sa présence qu'au tout début de l'exposition : «Bonjour! Ici Edgar Fruitier. C'est moi qui vous accompagnerai tout au long de l'exposition "Je vous entends chanter" » (Musée, 1996, p. 7). Les francophones du Québec risquent d'être familiers avec ce nom et cette voix, car Edgar Fruitier est non seulement un homme de théâtre, un artiste de la télévision et un animateur radiophonique bien connu, mais aussi un mélomane, un connaisseur et un collectionneur de musique classique réputé. Il occupe une position institutionnelle privilégiée au carrefour des médias dits de masse dont les auditoires représentent la clientèle-cible de l'exposition et des organisations culturelles dites savantes dont les publics forment l'une des plus fidèles clientèles des musées. En sa qualité de sujet énonciateur, il rend donc possible une double construction de l'exposition comme manifestation populaire et événement artistique, laquelle rejaillit sur le Musée de la civilisation ainsi posé à la fois comme espace public accessible et comme haut lieu d'éducation populaire et de culture.

Je doute cependant qu'il faille connaitre l'identité « réelle " du sujet parlant pour admettre la crédibilité du narrateur et comprendre l'étrange gravité des propos qu'il tient. Les textes constitutifs des mises en contexte sont tous prononcés sur un ton, à un rythme et selon un style caractéristiques d'un genre parlé qui, au sein de la sphère publique québécoise, connote d'entrée de jeu le privilège social, culturel et éducatif, si ce n'est économique. Combiné à l'omniprésence des constructions impersonnelles, ce trait ajoute à l'autorité assurée des textes et à la capacité qu'ils ont de désigner une réalité sur laquelle l'ici et maintenant de l'énonciation n'a apparemment pas de prise. Qu'il décrive l'apparition de la radio qui "abolit les distances et contribue à la création d'une culture musicale commune» (op. cit. p. 27) ou l'envahissement des foyers québécois par la télévision qui « révèle » à un vaste auditoire les talents et chansons d'ici » (idem), dans tous les cas, le narrateur s'efface au profit des faits apparemment indiscutables qui jalonnent l'histoire respective de la chanson et du Québec, posés comme deux entités closes sur elles-mêmes et ayant acquis leurs propriétés définitoires antérieurement aux textes qui les disent. En tant qu'objets discursifs et référents centraux des mises en contexte narrées, le Québec et la chanson sont effectivement construits comme des homologues, les destinées de l'un déterminant et reflétant à la fois celles de l'autre.

Les années 1970, c'est aussi la montée du nationalisme, de la contre-culture, de l'émancipation sexuelle et du féminisme. La chanson invite aux projets collectifs. Elle rassemble des milliers de Québécois sur le mont Royal ou les plaines d'Abraham pour célébrer la Fête nationale, la Superfrancofête ou la Chant'août. (op. cit., p. 69.)

Comme l'illustre cet énoncé, le passage entre chanson et société québécoise s'opère textuellement sans aucun recours aux marqueurs linguistiques conventionnellement utilisés pour signaler une transition (puisque, étant donné, alors que, etc.). L'absence quasi-totale de tels signes transitifs renforce le détachement du narrateur en regard de l'énonciation (Récanati, 1979) et contribue à naturaliser, voire à rendre évidente, l'étroite correspondance qui se trouve établie entre le domaine musical populaire et la sphère socio-politique au Québec.

À l'opposé, les commentaires des artistes regorgent d'une foule de marqueurs qui, tels les pronoms personnels, rendent indéniable la présence des sujets parlants. Cela permet, à mon sens, d'établir un lien entre le sujet énonciateur et son allocutaire et, dès lors, de créer un effet de subjectivité, sinon de partialité. La majorité des sujets qui prennent la parole sont des 
artistes à succès, devenus des figures clés de la scène publique locale par l'entremise de l'omniprésent appareil médiatique constitutif des domaines de la culture et du divertissement. Mais à travers les performances orales auto-réflexives auxquelles ils et elles se sont livrés en entrevue ${ }^{4}$, ces artistes sont positionnés d'abord et avant tout comme des individus offrant en partage leurs idées, leurs opinions et leurs souvenirs. Leurs propos sont organisés autour de chansons clés ou d'épisodes significatifs de leur carrière respective qui, transformés en autant de condensés d'expériences personnelles ou de moments de vie, servent de prétextes à la production d'un discours de l'intime où priment l'émotivité et l'introspection. Ainsi, invité à commenter le succès sans précédent qu'il remporta au milieu des années soixante, le chanteur populaire Michel Louvain se remémore les émeutes et l'hystérie collective que déclenchaient alors ses apparitions sur scène :

C'était fou... Vers la fin du spectacle, les filles essayaient d'monter sur la scène, de m'arracher mes vêtements... J'avais jamais vu ça !... J'avais des garde du corps ; ils m'ont fait sortir par en arrière, mais ça a pris la police pour que j'me rende jusqu'à l'auto. J'en revenais pas.

S'ils peuvent parfois convaincre, de tels énoncés me semblent davantage destinés à toucher, à émouvoir. Assise devant leurs photos respectives à écouter les commentaires de ces artistes, je dois d'ailleurs avouer m'être laissée prendre au charme de la complicité à laquelle ces textes invitent. Ils réussissent en effet à créer une atmosphère d'intimité qui, comme le suggèrent plusieurs remarques consignées dans le Journal des visiteurs, laissent peu de gens indifférents. Mais qu'on ne s'y méprenne pas! Ce discours de l'intime n'exclut pas le commentaire social ou politique. En effet, si les commentaires restent centrés sur l'expérience du sujet parlant, ils proposent souvent l'arrimage discursif de moments de vie de l'artiste à des moments de la vie sociale et politique du Québec. Le recours quasisystématique à des marqueurs de transition, ainsi qu'à des noms de lieux, de personnes ou d'événements datés, permet d'ancrer dans le lieu et le temps mêmes de l'énonciation, les rapports entre l'expérience individuelle d'une chanson et l'expérience collective de la communauté où elle s'inscrit, —qu'articulent ces textes parlés. "Poète de la ville... amoureux du pays» (Musée, 1996, p. 30), Raymond Lévesque décrit par exemple l'atmosphère qui régnait, au début des années soixante, dans les petites boîtes de la rue Crescent à Montréal où il présentait des compositions qui, comme Bozo les culottes, étaient de l'actualité politique mise en chanson ; "c'était injuste », «j'étais révolté », ajoute-t-il, en parlant des arrestations gratuites d'activistes et d'intellectuels soupçonnés, en 1963, d'être membres du Front de libération du Québec (FLQ) alors en émergence, et dont il raconte les actions et les destinées dans cette chanson.

Toute visite un tant soit peu attentive de l'exposition implique de passer d'une série de textes à l'autre, de se mouvoir dans un environnement sans cesse changeant au sein duquel alternent des voix personnelles et impersonnelles, des compte rendus factuels qui fournissent des informations générales ponctuelles et des propos empreints d'émotion qui relatent des expériences intimes. Mes observations m'incitent à penser que la complexité de l'espace sonore créé par ces voix (et les musiques) distinctes qui s'entrecroisent se laisse néanmoins relativement bien apprivoiser. En fait, une impression de cohérence se dégage peu à peu des allerretour entre les textes entendus du fait que la place de la musique populaire au Québec se trouve vraisemblablement définie à la jonction des lignes de forces totalisantes et individualisantes que construisent les mises en contexte et les commentaires : elle prend forme aux points de rencontre du micro et du macro, du particulier et du général, du personnel et du

4. Alors que les artistes semblent se livrer spontanément, en fait ils et elles répondent à des questions concernant quelques-unes de leurs chansons, choisies la plupart du temps par l'équipe du Musée. Toute trace de l'intervieweur a été effacée lors du montage.

5. Contrairement aux mises en contexte qui sont reproduites intégralement dans le Recueil des textes de l'exposition édité par le Musée (Musée, 1996), les entrevues commentaires n'ont fait l'objet d'aucune transcription officielle. J'en ai relevé des passages alors que j'écoutais les entrevues et c'est sur cette base que je me permets d'en citer des extraits. 
sociétal, de l'intime et du public - et non à l'un ou l'autre de ces pôles. Telle chanson est liée aux individus, à l'événement spécifique ou au contexte qu'elle dénote pour l'artiste l'ayant créée ou l'auditeur ou l'auditrice l'ayant appréciée ; tel genre musical est lié à quelque tendance plus ou moins récente sur une scène musicale donnée, qu'elle soit locale ou internationale ; tel album ou spectacle est lié à un moment précis du développement de l'industrie québécoise de la musique et, dès lors, à l'un desdits points tournants de l'histoire politique, économique ou sociale dudit peuple québécois. En plus de localiser simultanément la chanson québécoise sur une variété d'échelles, ces aller-retour contribuent à la définir comme une "présence à la plurialité des temps " (de Certeau 1990, note 7, p. 320), voire comme mémoire, selon l'acception ancestrale du terme. Chacun à leur façon, les textes constitutifs de l'exposition disent le Québec, les Québécois et la chanson québécoise en les faisant circuler à travers une multitude d'événements passés qui les constituent ; mais à la manière de la métis que problématise de Certeau, ces événements situés «suppute[nt] et prévoi[en]t aussi les "voies multiples de l'avenir" en combinant les particularités antécédentes ou possibles" (op. cit., p. 125).

La place de la musique populaire québécoise me semble produite par l'interaction de formes distinctes de mémoire que mobilisent en les construisant les deux séries de textes parlés/ narrés. D'une part s'y trouve articulée une forme collective et intégratrice de mémoire que les mises en contexte contribuent à officialiser en l'instituant. Elle est axée sur la trajectoire prétendument commune d'un peuple, de ses artistes et de sa musique, dont elle recherche les origines (sinon le moment originaire) de façon à y établir les fondements (sinon l'ontologie) de l'identité québécoise - comme s'il s'agissait d'un phénomène univoque et immuable. Se remémorer la musique populaire québécoise revient alors à établir son existence en tablant sur son authenticité, sa longévité et sa permanence dans le temps et dans l'espace en recourant, semble-t-il, à une forme d'essentialisme stratégique. D'autre part est aussi mise en cause une forme de mémoire individualisante que les commentaires participant à légitimer en la rendant généralisable. Celle-ci mise sur les trajectoires de stars héros qui, en vertu même de leurs particularités, sont aisément justiciables d'une interprétation littérale selon laquelle les événements, les objets et les émotions qui en sont constitutifs sont indépassables et intransitifs, étant significatifs en et par eux-mêmes uniquement. Mais, dans la mesure où les sujets énonciateurs sont d'abord construits comme des personnes relatant leurs expériences intimes, leurs trajectoires peuvent aussi s'avérer exemplaires et à ce titre, conserver leur singularité tout en se présentant comme «une instance parmi d'autres d'une catégorie plus générale» (Todorov, 1995, p. 30). Toute individualisante qu'elle soit, cette mémoire agit ainsi pour réactualiser les rapports (affectifs notamment) qui, médiatisés par une pratique culturelle donnée, lient le «passé présent » des expériences du sujet consommateur/amateur de musique populaire au " passé présent » de ses expériences en tant que sujet civil participant à la vie de la communauté québécoise dans son ensemble. Se remémorer la musique populaire québécoise revient alors à s'y identifier et à s'y reconnaître en tant que Québécois ou Québécoise.

Au sein de l'exposition, la musique populaire québécoise dite chanson est ainsi configurée simultanément à l'échelle de l'individu, de la population et de la société dont elle constituerait l'une des préoccupations communes, passée, présente et future. Par l'intermédiaire des formes de mémoire distinctes que mobilisent ses textes parlés/narrés, et dont les visées sont à la fois totalisantes et individualisantes, "Je vous entends chanter" semble bel et bien articuler la musique populaire comme l'« affaire de tout le monde ».

\section{« NOMMER » : LES LIEUX DE LA CHANSON}

Comme l'illustrent les débats souvent houleux qui entourent le recours sélectif aux qualificatifs tels que «Québécois », « francophones » ou « ethniques » pour identifier certains individus ou certains groupes, nommer est une opération stratégique qui permet à la fois de distinguer, de catégoriser, de classer et de sélectionner. En ce sens, il s'agit d'un processus performatif qui participe à l'attribution de la différence dans et par le discours. Comme l'indique Probyn, «le terrain de la différence est profondément marqué par les limites historiques 
qu'impose l'être-nommé» (Probyn, op. cit. p. 25, traduction L. G.), et ce, ajouterais-je, que l'êtrenommé mette en cause des individus, des événements ou des choses. En examinant comment l'exposition nomme la musique populaire québécoise, plus particulièrement les regroupements d'objets et de sujets effectués pour rendre compte de ce champ, je me propose d'interroger les frontières contingentes qui lui sont imposées et qui, ce faisant, rendent possibles certaines formes d'allégeance ou d'appartenance à « la culture québécoise ».

Les noms attribués aux principales zones de « Je vous entends chanter » sont « chanson populaire ", "chanson rock» et "chanson country ". Par le biais des adjectifs « populaire ", « rock " et « country », lesquels évoquent une catégorisation internationalement admise qui fait du genre le principe classificatoire par excellence en musique populaire, ces appellations permettent de distinguer les pratiques et les produits constitutifs de l'exposition de ceux dénommés " classiques », tout en marquant leur appartenance à un domaine clé de la culture et du divertissement contemporains dont les frontières débordent largement celles du Québec. Par le biais du mot «chanson », elles contribuent à souligner le caractère résolument francophone de l'être-nommé qui se voit ainsi apparenté à des traditions musicales originaires de la France mais qui, dans la foulée de la colonisation, se sont aussi développées ailleurs en Europe, en Amérique, et aux Antilles notamment (Baillargeon et Côté, 1991 ; Rioux, 1992). En joignant systématiquement les qualificatifs de genre au mot « chanson », un terme possédant par ailleurs des connotations complexes au Québec, les appellations retenues permettent surtout, selon moi, de signifier qu'il s'agit non seulement de musique populaire francophone, mais bel et bien de musique québécoise, avec ses traditions et son histoire propres?.

Différents points de vue de l'usage linguistique (Granger, 1988) circonscrivent, me semblet-il, les significations concurrentes du terme « chanson " dans le contexte québécois contemporain. Du point de vue de la composition ou de la production, le terme dénote une pièce musicale comprenant des paroles : on parle ainsi de la création ou de l'interprétation d'une chanson, quel qu'en soit le genre ou le style. Du point de vue de l'analyse, historique notamment, le mot signifie plutôt un genre ou un mouvement musical au sein duquel les paroles, obéissant aux règles de la poésie à formes fixes (Chamberland et Gaulin, 1994), occupent une place prépondérante : il est ainsi question de la chanson française ou, plus généralement, de la chanson à texte. Au Québec, une forte coloration politique a été attachée à cet usage. En effet, l'expression « la chanson québécoise » a longtemps servi à désigner exclusivement le répertoire et les pratiques des chansonniers des années soixante, c'est-à-dire un ensemble d'auteurs-compositeurs-interprètes dont les créations témoignaient de leur engagement sociopolitique et de leur parti pris gauchisant, souvent nationaliste, sinon séparatiste. L'intervention de différents agents de consécration, dont les institutions médiatiques et littéraires, a contribué à promouvoir « la chanson " conçue, dans ce contexte, non seulement comme un genre influent, mais bien comme le seul genre authentiquement québécois (par comparaison aux autres genres d'inspiration étatsunienne) et, dès lors, porteur de la seule musique qui puisse se réclamer comme culturellement significative et représentative du « peuple québécois ». Depuis le début de la présente décennie, un troisième point de vue a fait surface, que je qualifie d'industriel en référence au milieu au sein duquel il a d'abord été articulé (Grenier, 1994). L'étroite association entre chanson et culture québécoise est préservé, mais non la forte connotation politique qui accompagne souvent l'usage du terme : ce dernier constitue le nom commun attribué à toute musique populaire créée ou produite au Québec, et ce, indépendamment des distinctions de

6. Il est à noter que le discours public dont la musique populaire fait l'objet au Québec a déjà recours à cette terminologie. Cependant, la notoriété du musée en tant qu'institution culturelle, combinée au prestige du Musée de la civilisation de Québec en tant qu'institution nationale dynamique et avant-gardiste en officialise davantage l'usage, et contribue à légitimer les classifications et les ordonnances qu'elles instaurent.

7. Dans le Guidebook (Musée, 1995) mis à la disposition du public visiteur non francophone, les zones sont nommées popular music, rock music, country music - une traduction qui tend à la fois à évacuer les connotations complexes qui accompagnent l'usage du mot « chanson " au Québec et à minimiser l'importance que les appellations françaises contribuent à donner à l'ancrage local, sinon national, des pratiques et des produits musicaux qui sont l'objet de l'exposition. 
genre ou de style. Parler de la chanson québécoise d'un tel point de vue, c'est désigner l'une ou l'autre des multiples pratiques musicales qui coexistent sur le territoire québécois et, dans le même temps, recourir à une étiquette générique qui signifie le Québec comme un espace musical pluriel mais néanmoins consolidé (Grenier, 1993).

La façon dont le Musée nomme les principales zones de « Je vous entends chanter » me semble fortement orientée par ce point de vue dit industriel. La nomenclature retenue permet en effet de mettre de l'avant un large éventail de produits et de pratiques musicaux, tout en accentuant leur égale contribution à la formation et au maintien d'une culture musicale proprement québécoise. Quoique le titre de l'exposition évoque le mouvement chansonnier (il est emprunté à la célèbre chanson Les gens de mon pays créée par Gilles Vigneault, l'un des principaux poètes défenseurs du Québec en musique), son être-nommé se veut différencié, en soulignant les distinctions de genres et de styles, mais aussi inclusif, en insistant sur leur commune participation au domaine musical populaire du Québec. Comme l'ont unanimement souligné les journalistes, cette façon de faire contraste singulièrement avec le discours dominant, au sein duquel la chanson à texte définit, en la résumant, une «chanson québécoise » prétendument née à la fin des années cinquante.

« Je vous entends chanter » nous fait prendre conscience de la diversité de l'expression chantée du Québec. Les concepteurs ont laissé de côté les récits conventionnels et officiels qui font tout démarrer avec Félix ${ }^{8}$. Toutes les époques de la chanson populaire y sont représentées. [...] La catégorisation choisie par les organisateurs se veut totalement affranchie du syndrome de la fleur de lis ou de la ceinture fléchée. Le country, le western et le rock ont leur place. (Robitaille, 1995, p. A-1.)

L'exposition n'émet aucun jugement sur l'authenticité, voire le degré de québécitude de tel genre musical ou de telle pièce musicale, mais elle fait néanmoins appel à certains des critères de valorisation constitutifs de l'usage analytique du terme chanson. Une importance prépondérante est accordée aux auteurs-compositeurs-interprètes, au détriment des interprètes, tendance qui va de pair, me semble-t-il, avec le fait de privilégier les chansons à «message » - dont les paroles, écrites dans une langue correcte et suivant les formes poétiques d'usage, seraient significatives - au détriment des chansons dites «à danser " ou qui ne visent qu'à divertir'. Qui plus est, quoique sa parenté avec un nationalisme d'allégeance séparatiste soit mise en sourdine, la chanson québécoise nommée par le Musée demeure d'abord et avant tout le média privilégié de célébration de la fierté nationale, voire « le point de vue privilégié pour comprendre l'identité québécoise » (Musée, 1995, p. 2). L'identité, plus précisément la quête d'identité des Québécois francophones par la chanson québécoise, constitue en effet le fil conducteur de la thématique mise de l'avant par les responsables de l'exposition. Reconnaître la chanson dans toute sa diversité signifie reconnaitre les multiples voies (et voix) par lesquelles cette quête prétendument commune à tous les genres et les styles a pris forme en paroles et en musiques. Contribue d'ailleurs à cette articulation de l'être-nommé-chanson le recours à Claude Dubois, un artiste reconnu tant pour son engagement politique que pour sa polyvalence musicale, comme président d'honneur de l'exposition et maittre de cérémonie lors de l'inauguration.

8. Félix Leclerc est ce poète et auteur-compositeur-interprète mort en 1988 qu'une majorité de critiques et d'analystes considèrent comme le père du mouvement chansonnier des années soixante et, dès lors, l'instigateur d'une chanson proprement québécoise. Marque du caractère légendaire attribué au personnage, Félix est le nom des trophées que remet l'Association de l'industrie du disque, du spectacle et de la vidéo du Québec (ADISQ) lors de son gala annuel destiné à célébrer le talent et le succès des artistes québécois.

9. François Desmeules, membre de l'équipe responsable notamment de la sélection musicale, a d'ailleurs reconnu l'existence d'un « léger parti pris » : «Il y a un intérêt marqué pour les auteurs-compositeurs-interprètes... Alors à partir de là évidemment, on a élagué. Il y a, je dois l'admettre aussi, un léger parti pris pour éviter, comment dire... Il y a des choses qui sont drôles, il y a des choses qui sont kitsch, mais passé un certain stade, il y a des choses qui sont simplement pas très intéressantes et qui, parfois, ont quand même plu dans les époques, un peu, de sécheresse, là, culturelle. Et on a un peu délaissé ça. Je pense pas qu'on ait tellement illustré la période de la chanson disco au Québec, qui était d'ailleurs colorée d'anglicismes. » (Radio-Canada International, transcription par Caisse et Chartier, 1996, p. 4.) 
« Un cri, un appel, une déclaration d'indépendance » lance Claude Dubois, en entrevue. En retrouvant son costume de scène bleu métallique d'il y a vingt ans et des poussières, à côté de la guitare de Félix, de l'harmonica de la Bolduc ${ }^{10}$, des maracas d'Alys Robi ${ }^{11}$ et des vestes à franges de Willie Lamothe ${ }^{12}$, Claude Dubois a eu un choc. « J'ai senti la pulsion d'une petite nation à l'écart, la voix unique d'un peuple coincé. La chanson est au Québec ce que le reggae est à la Jamaïque! Les peuples mal pris produisent souvent des musiques originales! » (Tremblay, 1995, p. B-4.)

Dans le cadre de l'exposition, les chansonniers ne constituent donc pas la seule incarnation de l'artiste québécois ayant à cœur la destinée du Québec et de son " peuple ». Les artistes associés aux différents genres musicaux représentés au Musée sont également construits comme des citoyens engagés, quoique de manière différente. Leurs préoccupations de citoyens sont évoquées par le biais des décors spécifiques, destinés à reconstruire l'atmosphère, sinon l'espace physique jugé caractéristique du genre auquel l'artiste est associé, au sein duquel leurs objets, leurs photos, leurs enregistrements et, le cas échéant, leurs commentaires sont situés. Ainsi, la zone consacrée aux poètes-chansonniers recrée l'atmosphère typique des boîtes à chansons : de petite cafés intimistes où des artistes interprètent, souvent en solo, des compositions célébrant le pays à construire ou s'attaquant à quelque problème social, devant un auditoire relativement jeune censément porté par les mêmes aspirations au changement. Plusieurs chansonniers de renom sont aussi au centre d'une zone dédiée aux rassemblements populaires des années soixante-dix ; y sont présentés des extraits de spectacles à grand déploiement qui, dans le cadre de la Fête nationale du Québec notamment, réunissaient sur une même scène extérieure quelques-uns des « grands noms » du moment, acclamés par des dizaines de milliers de personnes. Quant au décor privilégié pour dépeindre la chanson country, loin de recourir aux éléments caricaturaux associés au genre (les chevaux, les grands espaces et les rodéos), il met l'accent sur la ville moderne : les artistes y sont présentés comme de "nouveaux cowboys » qui, grâ.ce à la sincérité et à la simplicité de chansons qui «viennent du cœeur et s'adressent au cœur» (Musée 1995, p. 133) et qui parlent d'amours malheureuses, d'éloignement, de solitude et de problèmes familiaux, font des valeurs traditionnelles du monde rural une composante à part entière de la vie urbaine contemporaine. La scène des salles de concert apparaît comme le lieu de prédilection de la chanson rock, cette " musique primaire et sensuelle (Musée, 1995, p. 165) qui, combinée à des textes d'" une profondeur qu'on ne retrouve pas dans le rock anglophone 》 (idem), attire les rebelles et repousse les éléments plus conservateurs de la société québécoise. Enfin, les médias électroniques constituent le décor par excellence des chanteurs et chanteuses pop : plusieurs de ces artistes sont présentés sur le plateau de tournage des émissions de télévision qui, en leur permettant de participer à la vie quotidienne de tant de foyers québécois de différents milieux, ont contribué à les consacrer vedettes.

Ce que l'exposition nomme chanson se trouve ainsi défini au carrefour de cafés, de scènes extérieures, de salles de concert, de la ville et de la campagne, du chez-soi et des médias. Évoquant des formes de subjectivité civique et culturelle distinctes, de même que certains espaces privilégiés de leur articulation, ces différents décors me semblent constituer les principaux « lieux de mémoire " de la musique québécoise construite par le Musée. Dans la foulée des travaux de l'historien Pierre Nora, j'utilise cette expression pour désigner des lieux à caractère matériel, fonctionnel et symbolique, dont la raison d'être est « de bloquer le travail de l'oubli, de fixer un état de choses [...] de matérialiser l'immatériel pour [...] enfermer le maximum

10. Nom de scène de Mme Édouard Bolduc, née Mary Travers, célèbre auteure-compositeure-interprète des années vingt et trente dont les chansons liaient des musiques de facture traditionnelle à des textes traitant souvent de sujets d'actualité dans un langage populaire.

11. Vedette de cabaret et chanteuse populaire qui a connu ses plus grands succès dans les années 40 en interprétant notamment des chansons d'inspiration latino-américaine, dont la célèbre Tico-Tico dont elle a traduit les paroles.

12. Interprète et auteur-compositeur, il est l'un des pionniers de la musique de type country-western. Sa carrière atteint son apogée dans les années soixante-dix alors qu'il anime une émission de télévision extrêmement populaire qui contribue à la diffusion de ce genre musical longtemps boudé par les médias à grand public, "Le ranch à Willie ». 
de sens dans un minimum de signes $\gg$ (Nora, 1984, p. xxxv). À titre d'exemple, il est possible de considérer l'émission « Jeunesse d'aujourd'hui » comme un lieu matériel dont les propriétés sont relatives aux conditions technologiques de production caractéristiques des débuts de la télévision privée au Québec; qui a entre autres pour fonction d'assurer la diffusion et la promotion d'artistes et de produits musicaux; et qui symbolise particulièrement la musique « jeune » des années soixante, celle d'une génération montante vibrant au rythme de la culture populaire américaine. Refermés sur leur identité propre, mais ouverts sur l'étendue des significations dont ils sont investis, ces lieux permettent de désigner des fragments d'un passé vécu qui entraînent dans leur sillage toute une panoplie d'images, de sons et de situations familières dont ils sont solidaires dans les souvenirs qu'ils éveillent. Ainsi, voir et entendre des extraits

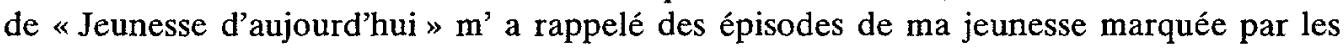
musiques d'artistes pop que me faisaient découvrir mes sœurs aînées, qui m'ont fait me remémorer mon enfance dans le quartier Ahuntisc de Montréal et toute la gamme des émotions et des souvenirs qui s'y rattachent. Qui plus est, comme le souligne Nora, contrairement aux autres signes mémoratifs, les lieux de mémoire sont le fruit d'une interaction où mémoire et histoire se déterminent réciproquement. Les lieux construits par « Je vous entends chanter » désignent aussi les fragments d'une histoire collective qu'ils contribuent à reconstituer en articulant des expériences musicales à des épisodes du passé culturel, linguistique, politique et économique du Québec et de son « peuple ». « Jeunesse d'aujourd'hui » évoque ainsi cette étape marquante de l'histoire de la musique populaire au Québec où une partie de la jeunesse a renoué avec la chanson francophone et où une industrie locale a commencé à s'établir ; ce faisant, elle est susceptible de rappeler aux visiteurs et visiteuses l'importance du développement de la langue française comme pilier d'une identité proprement québécoise (non plus canadienne-française) et du contrôle des leviers de l'économie par des intérêts québécois (non plus étrangers) deux revendications clés de la Révolution tranquille. L'invitation - l'obligation dirait Nora (op. cit., p. xxxi) - est ainsi faite à chaque sujet, visiteur ou participant, de se souvenir, mais d'une manière telle que sa propre mémoire privée permette le recouvrement de son appartenance à la collectivité québécoise, vue comme principe d'identification.

Une telle articulation de l'être-nommé-chanson procède donc d'une série de lieux dont la singularité n'est pas tributaire de leurs frontières spatiales et temporelles respectives, mais plutôt d'expériences, de relations et de connaissances spécifiques, médiatisées ici par des mémoires mises en histoire. Les formes concurrentes de chanson constitutives de la musique québécoise sont ainsi localisées dans des lieux qui sont d'ores et déjà liés à des expériences privées et à des mémoires collectives, bref, des passés préexistants que l'exposition concourt à réactualiser, à rendre présents. Les artistes, les produits musicaux et les objets personnels regroupés sous chacun de ces lieux de mémoire agissent, chacun à leur manière, comme médiateurs entre les passés « présents » d'individus ou de groupes particuliers participant au rock, à la musique pop ou au country et le passé "présent " du Québec et du peuple québécois. Bien qu'ils mettent en évidence des figures contrastées de la vie musicale présente et passée au Québec, les lieux de mémoire qui articulent la chanson québécoise tendent en effet à subsumer sous une histoire culturelle « nationale » unique les parcours singuliers des individus et des groupes dont les pratiques respectives ont rendu possible l'émergence et la consolidation d'une musique québécoise.

Comme l'écrivait un critique :

L'histoire de la chanson racontée au Musée, c'est l'histoire de toutes les chansons [...] C'est aussi l'histoire d'un peuple qui a porté et aimé ces chansons pour toutes sortes de raison - pour remercier le bon Dieu de nous donner un ciel si bleu, pour pardonner à toutes les blondes du monde, mais aussi pour affirmer une identité collective. Parions que ce n'est pas une coïncidence si l'exposition a commencé sa carrière en période référendaire. (Lepage, op. cit., p. D-9.)

La musique populaire devient ainsi une affaire politiquement importante dans la mesure où elle établit un pont entre les passés « présents » de communautés distinctes des sujets 
«JE ME SOUVIENS » ... EN CHANSONS : ARTICULATIONS DE LA CITOYENNETÉ CULTUFELLE ET DE L'IDENTTTAIRE DANS LE CHAMP MUSICAL POPULAIRE AU QUÉBEC

consommateurs/producteurs comme citoyens et une histoire unique qui est celle du « peuple québécois " que forment ces communautés réunies.

\section{« RACONTER » : ENTRE LA CHRONIQUE ET LE RÉCIT D'UNE RÉUSSITE}

Comme l'indique le sous-titre de l'exposition, le public visiteur de « Je vous entends chanter » est convié à découvrir « 100 ans d'histoire de chanson québécoise ». Bien qu'il vise à présenter les «faits » qui ont marqué ladite évolution de cette musique, le panorama offert n'a rien de l'exposé didactique. L'objectif étant de divertir tout en favorisant l'acquisition de connaissances par le biais d'un contenu qui puisse intéresser et informer en suscitant l'émotion (Bilodeau, 1994), les responsables ont opté pour ce mode d'exposition éprouvé qu'est le récit. Considérant la popularité des récits en tout genre qui forment la matière même de nombreux produits culturels de grande consommation au Québec (film, best-seller, téléroman, etc.), le recours à la narration comme stratégie d'exposition n'est guère étonnant - d'autant plus que ce choix émane d'une institution pour laquelle la communication constitue le parti pris avoué de ses pratiques muséologiques (Arpin, 1992). Mais quelles que soient les raisons qui la motivent, le déploiement de cette stratégie, dans un contexte où la narration médiatise la reconstitution et la diffusion d'un savoir historique « positif " plutôt que la création d'une œuvre de fiction, tend à produire un effet singulier, que celui-ci soit explicitement recherché ou non. L'exposition propose une histoire de la chanson québécoise au sein de laquelle le hasard, les coïncidences et la contingence n'ont aucune place : comme dans un récit bien ficelé, aucune piste ne reste embrouillée et aucun événement ne demeure inexpliqué, toute chose a sa raison d'être et toute conséquence a sa cause. Serait-ce là la condition nécessaire pour garantir la véracité des « faits » relatés et assurer la crédibilité de la représentation proposée en tant que discours historique proprement dit? Les arguments développés par Hayden Whyte concernant la valeur de la narrativité en histoire m'incitent à le penser : la production d'un discours narratif permettrait de créer une représentation singulière des événements " réels » qui « affiche la cohérence, l'intégrité, la complétude et la finitude d'une image de la vie qui est et ne peut être qu'imaginaire » (Whyte, 1987, p. 24, traduction L.G.).

C'est par l'entremise de deux narratifs distincts mais superposés que l'exposition articule, en la racontant, la place de la musique populaire québécoise. Le premier narratif est caractérisé par la prédominance de la chronologie comme principe organisateur du discours. L'histoire de la musique populaire qu'il produit prend la forme d'une chronique : une série d'événements exposés selon une séquence visant à reproduire l'ordre dans lequel ils se sont passés ou, plus exactement, dans lequel ils ont été remémorés. Ce faisant, non seulement la " réalité " des événements mis en cause par le narratif est fondée, mais le caractère proprement historique du récit se trouve légitimé. Comme l'explique White :

Ces événements [du discours historique] sont réels non pas parce qu'ils se sont produits mais bien parce que, premièrement, on s'en est souvenu et, deuxièmement, ils peuvent trouver une place dans une séquence chronologiquement ordonnée. Pour que leur exposé soit considéré comme un exposé historique, il n'est cependant pas suffisant qu'ils soient rapportés dans l'ordre de leur séquence originale. C'est le fait qu'ils peuvent être rapportés autrement, dans l'ordre d'un narratif, qui les rend à la fois douteux quant à leur authenticité et susceptibles d'être considérés comme des signes de réalité (ibid, p. 20).

«Je vous entends chanter " découpe cette histoire en une série de chapitres mutuellement exclusifs. Celle-ci débute à la fin du XIX ${ }^{e}$ siècle, alors que des milliers de Québécois et de Québécoises de langue française, désireux de profiter des occasions d'emplois créées par une révolution industrielle en plein essor, quittent les campagnes pour s'installer massivement dans les villes naissantes. Si cette vague de migration est vue comme représentant les « racines »du Québec moderne, les chansons folkloriques qu'interprétaient les premières générations de citadirıs et citadines sont construites, quant à elles, comme les « racines » de la musique populaire québécoise. Le récit s'attarde ensuite, en bloc, aux cinq premières décennies du présent siècle, en soulignant particulièrement l'apport de la radio à la création d'une culture commune, 
à une époque largement dominée par les musiques françaises et américaines. Suit un chapitre consacré aux années soixante, où est mise en scène une jeunesse friande de rock'n'roll mais qui, grâce à l'arrivée de la télévision, renoue avec la chanson francophone interprétée par des vedettes locales copiant les succès américains de l'heure. L'histoire de la décennie suivante, qui marque l'« âge d'or de la chanson québécoise », présente des événements qui témoignent de la participation de la musique populaire aux projets collectifs en "une époque opulente qui fait la fête à la diversité et à la nouveauté" (op. cit., p. 69). Le tout prend fin par le récit des faits marquants des décennies quatre-vingt et quatre-vingt-dix, caractérisées respectivement par le désenchantement qu'accompagnent une importante crise des valeurs et un regain de vitalité que même une situation économique difficile ne semble pas pouvoir freiner. La chronique se termine en proposant un regard sur les événements actuels sans toutefois offrir de réelle conclusion - ce qui incite à penser que l'histoire n'est pas finie, que tout comme la société québécoise et le peuple québécois, la musique québécoise continuera sans cesse de se développer.

Le second narratif constitutif de l'exposition n'est pas tant gouverné par une chronologie que par une intrigue, soit une combinaison d'événements «dotés de sens en étant identifiés comme des parties d'un tout intégré " (White, 1987, p. 24, traduction L. G.). À l'instar de nombreuses fictions de grande consommation circulant au Québec (Saint-Jacques et al., 1995), le Musée propose un récit de la réussite dont l'intrigue est axée sur les obstacles que doit surmonter le personnage central pour atteindre son but. «Je vous entends chanter » raconte en quelque sorte les tribulations de deux principaux personnages, une chanson en quête de sa propre identité et un peuple en quête de lui-même, dont elle présente les parcours de manière analogue. L'exposition raconte comment la chanson québécoise a réussi à se distinguer comme forme musicale québécoise à part entière malgré des obstacles tels la petitesse du marché, l'omniprésence de produits concurrents, le développement tardif des infrastructures industrielles locales et le manque de ressources financières adéquates mises à la disposition de ses créateurs et créatrices. Mais elle raconte aussi, dans le même temps, comment les Québécois et les Québécoises ont réussi à s'affirmer comme peuple après une longue et difficile lutte pour maintenir et promouvoir la langue française, pour résister à l'impérialisme culturel et au colonialisme interne, pour survivre aux sévères récessions économiques et, ce faisant, pour vraiment devenir la « société distincte " que le Québec aurait soi-disant toujours été.

Au sein de ce récit, la réussite obtenue par les protagonistes est attribuée à l'amour du Québec francophone pour «sa chanson » (Musée, 1996, p. 7) et à la fidélité que témoigne la chanson québécoise à l'endroit du peuple dont elle refléterait les aspirations profondes - deux éléments qui ont servi de pivots à la conception même de l'exposition :

Il est très important de comprendre à quel point, au Québec, la chanson est un art majeur qui ne doit son épanouissement qu'à l'amour de son public. Comment expliquer autrement le fait qu'une industrie du disque et du spectacle ait pu se développer dans une société si peu peuplée. Les Québécois francophones se sont toujours reconnus dans leur chanson et lui sont restés fidèles parce qu'elle leur est restée fidèle. La thématique de l'exposition s'enroule autour de la quête de son identité par la chanson québécoise. Cette quête est intimement liée à celle, simultanée, de toute la société québécoise dont la chanson est le reflet fidèle. (Bilodeau, 1994, p. 10.)

Les mises en contexte du narrateur et les entrevues commentaires des artistes, sur lesquels je me suis penchée dans la première partie de cette analyse, constituent les principaux textes parlés au sein desquels s'élabore textuellement le récit d'une réussite fondée sur l'amour et la fidélité. Mais, selon moi, les assemblages d'objets personnels appartenant aux artistes contribuent à ce narratif ${ }^{13}$ puisqu'ils peuvent être vus comme autant de preuves tangibles de la réussite ici présentée sous la forme du succès et, à ce titre, attester du dénouement heureux de l'intrigue. Supports visuels à la catégorisation par genre, ces objets servent à reconstituer les

13. L'impressionnante quantité d'objets recueillis a forcé les responsables à placer certains artefacts en rotation. Je réfère ici aux objets présentés lors des mois de janvier et février 1996, au cours desquels j'ai effectué mes observations. 
«JE ME SOUVIENS "... EN CHANSONS : ARTICULATIONS DE LA CITOYENNETÉ CULTURELLE ET DE L'IDENTITAIRE DANS LE CHAMP MUSICAL POPULAIRE AU QUÉBEC

lieux respectifs des formes concurrentes de la chanson constitutives de la musique populaire au Québec; mais ils permettent aussi d'esquisser les trajectoires de carrière contrastées des artistes qui ont contribué à l'éclosion d'une chanson proprement québécoise. Évoquant tant la popularité d'un artiste donné que celle du genre particulier de chanson québécoise qu'il ou elle incarne, les artefacts constitutifs de « Je vous entends chanter » agissent comme autant de marqueurs des figures distinctes du succès qui prévalent dans le domaine musical populaire au Québec et, ce faisant, des différentes économies de valeur (Grossberg, 1992), politiques, commerciales, esthétiques ou affectives, qu'elles articulent.

L'exposition met en évidence cinq principales figures du succès. Le succès apparaît comme l'évaluation d'une performance commerciale d'un produit musical sur les marchés : le disque d'or reçu par le chanteur Marc Hamilton, qui souligne la vente de plus d'un million de copies du 45 tours Comme j'ai toujours envie d'aimer est la marque d'une telle performance. Le succès signifie aussi l'estime ou la reconnaissance par les pairs dont témoignent, notamment, les trophées Félix décernés par l'Association des industries du disque, du spectacle et de la vidéo du Québec (ADISQ) prêtés par Pierre Flyn (microsillon rock 1988) et George Hamel (microsillon country/western 1991). Une troisième figure identifie le succès à la célébrité ou à la popularité qu'acquiert un artiste auprès du public consommateur ou auditeur : une étape importante de la carrière de Ginette Reno est ainsi évoquée par le Trophée découverte de l'année Écho-Vedette que lui ont décerné les lecteurs et lectrices de ce magazine artistique à grand tirage en 1964. L'estime vouée à un artiste par une communauté donnée constitue une quatrième figure : des objets comme le doctoral honorifique qu'a octroyé l'Université Laval au parolier Luc Plamondon et le certificat de désignation de nom de rue offert à Michel Louvain par la Ville de Thetford Mines en 1988 jouent un tel rôle. Enfin, le rayonnement extérieur, voire lat renommée que s'est acquise un artiste sur des scènes culturelles autres que québécoises, est une autre figure de succès qui se traduit matériellement par des objets tels que le costume de scène porté par René Simard lors du Festival international de la chanson de Tokyo en 1974, le dépliant publicitaire Tycoon 1994 qui assurait la promotion de l'adaptation anglaise de l'opéra-rock Starmania aux États-Unis et le programme-souvenir du spectacle offert par Gilles Vigneault à l'Olympia de Paris en 1984.

La majorité de ces objets n'ont pas acquis leur statut de marqueurs de succès par leur seule entrée au Musée. Les trophées, prix, médailles et distinctions de toutes sortes occupent déjà une place de choix au sein d'une scène musicale qui, au fil des ans, a su développer un ensemble d'outils culturels adaptés, permettant non seulement de signaler le succès mais d'en déterminer les critères pertinents. Le succès ne constitue pas, en effet, une réalité installée une fois pour toutes mais plutôt une réalité en constant changement. Produit du mouvement d'industrialisation endogène de la musique populaire au Québec, il est tributaire d'un ensemble particulier de stratégies d'individus, d'entreprises et d'organisations qui mettent à profit un certain nombre d'instruments de mesure qui, tels les palmarès et les scrutins, servent à évaluer les effets de ces stratégies ; ces instruments forment une partie intégrante des rituels de valorisation, c'est-à-dire des processus institutionnalisés comme les galas, les festivals et les concours, au cours desquels ils servent à promouvoir, en les hiérarchisant, certains produits musicaux, certaines pratiques musicales et certains artistes (Grenier, Deglise et Martin, 1996). Le fait d'être exposés dans le cadre de «Je vous entends chanter » confirme la légitimité et ajoute peut-être même à la valeur culturelle non seulement des objets, mais aussi des instruments de mesure et des rituels de valorisation dont ils sont solidaires. Il est à noter qu'aucun des textes de l'exposition ne lie explicitement ces objets aux agents industriels qui ont contribué à les ériger en signes d'un « vrai » succès, d'une « réelle » réussite. Le rôle clé joué par la consolidation de l'industrie musicale locale est mentionné mais non l'apport singulier des individus et des entreprises qui en sont responsables. Le narratif met plutôt à l'honneur l'industrie musicale dans son ensemble, en la présentant comme un cas exemplaire de l'« entrepreneurship » culturel au Québec. Si l'on se fie à l'histoire racontée par le Musée, exercer le contrôle économique sur la musique signifierait détenir le contrôle sur ce qu'elle exprime ; dans une telle 
optique, la réussite de l'industrie peut aussi être construite comme celle du peuple québécois dont elle produit « la » musique.

Bien entendu, à l'instar des souliers de ballet portés par la chanteuse Michèle Richard dans les années cinquante ou de la statuette porte-bonheur grigri rapportée par Richard Séguin d'un voyage en Équateur, tous les biens personnels prêtés par les artistes ou leurs proches ne relèvent pas de cette catégorie d'objets. Tous possèdent une certaine valeur, sinon commerciale à tout le moins culturelle et affective, dans la mesure où ils incarnent matériellement le rapport singulier entre un artiste et son public qui définit la vedette, la star. Ces objets contribuent moins à désigner des moments de la vie privée des individus qui en sont les détenteurs légitimes qu'à recréer un épisode de la carrière d'artistes-vedettes qui, en tant qu'articulations singulières du star-système dont ces vedettes sont le produit, deviennent eux-mêmes et ellesmêmes quelque chose que possèdent les sujets auditeurs et consommateurs. Comme l'explique Frith, "[C]'est la forme marchande de la musique qui rend possible ce sentiment de possession musicale, mais ce n'est pas seulement le disque que les gens pensent posséder: nous sentons que nous possédons aussi la chanson elle-même, la performance particulière et l'artiste qui l'offre. En "possédant" la musique, nous en faisons une partie intégrante de notre identité et l'intégrons à notre expérience de soi » (Frith, 1987, p. 143, traduction L. G.). Par l'entremise de divers objets fétiches, le succès des artistes peut ainsi être construit comme étant aussi celui des sujets consommateurs/auditeurs et des communautés interprétatives qui les ont rendus populaires, et le cheminement des premiers comme étant le symbole du parcours des seconds.

Les centaines d'objets que réunit «Je vous entends chanter » réveillent eux aussi bien des souvenirs chez les visiteurs et visiteuses. Mais plus que de simples déclencheurs de mémoire, ils constituent les traces matérielles sur lesquelles reposent une mémoire-archive proprement musicale que l'exposition contribue à instituer. Reprenant à mon compte l'argument de Nora, j'entends par là «la constitution gigantesque et vertigineuse du stock matériel de ce dont il nous est impossible de se souvenir, répertoire insondable de ce dont nous pourrions avoir besoin de nous rappeler » (Nora, 1984, p. xxvi), et ainsi, reconstituer en objets le souvenir de la musique populaire québécoise.

La mise sur pied de l'exposition a nécessité beaucoup d'efforts de la part de l'équipe responsable de la conservation qui, habituée à travailler dans le cadre de prêts entre musées, a eu à solliciter des individus. Cette longue et ardue « chasse aux objets » n'avait pas pour objectif de recueillir un ensemble d'artefacts prédéterminés mais plutôt de découvrir, chez les artistes volontaires, des objets qui pourraient servir et dont ils ou elles accepteraient de se défaire gratuitement, pour une période de temps déterminée. Les médias ont fait grand cas de cette entreprise - d'autant que les principaux intéressés ont manifesté leur étonnement devant une telle initiative (entrer au musée de son vivant n'étant pas chose commune), devant la nature du processus de recherche, et des « découvertes » auxquels il a donné lieu. Considérons par exemple l'échange suivant, extrait d'une émission diffusée sur les ondes d'une station radiophonique de la ville de Québec:

Animatrice :[...] Donc tout ce que le Québec compte d'artistes a vu débarquer chez eux de petits découvreurs en gants blancs.

Jean-Pierre Ferland: Ils sont venus chez moi avec des gants blancs, pour vrai, en me disant : «Qu'est-ce que vous voulez nous donner pour le... » Faque j'avais ma guitare douze cordes sur laquelle j'avais composé Le petit roi ; j'ai dit : « Ça fais-tu votre affaire ? » Alors je lui ai donné. Après ça, j'avais une paire de pantalons dans le temps où j'étais love and peace, avec des pattes d'éléphant en cuir et des petites roses un peu partout, je lui ai donnée. Et je lui ai donné la chemise que Léo Ferré m'avait donnée, sa chemise rouge qui était sa chemise de révolutionnaire. Puis ils m'ont demandé de donner des textes de chansons, et, j'en avais pas parce que je jette tout. Dubois : Moi d'abord je les ai trouvés tellement respectueux, t'sais parce qu'ils se promenaient dans mon sous-sol avec des gants blancs, t'sais, puis ils remontaient avec des bouts de papiers que j'avais écrits il y a des années, en me disant : " Ah mon Dieu ! mais ça, faut préserver ça... » Et... bon moi, je n'ai jamais pensé à ça... parce qu'évidemment y avait des textes qui ont 
jamais été publiés, y ont retrouvé ça, et puis ils se promenaient avec des gants blancs, comme ça, avec des bouts de papier, des bouts de chiffons... (Caisse et Chartier, 1995, p. 3)

Les objets « retrouvés » ne doivent pas leur sauvegarde à la seule bonne volonté ou au narcissisme plus ou moins avoué des artistes, mais bien aux conditions matérielles qui permettaient à ces derniers d'accumuler ou pas ces multiples petites choses qui semblent parfois banales, mais qu'on se refuse souvent à jeter.

$\mathrm{Cr}$, il apparut bien logiquement que les artistes mariés ou possédant une maison avec sous-sol étaient ceux qui avaient conservé le plus de souvenirs. «Pourquoi la majorité des objets ayant appartenu à Beau Dommage viennent de chez Réal Desrosiers ? Parce qu'à cette époque il était le seul à avoir une maison pis un soul-sol », répond mon interlocuteur (François Desmeules), » (Tremblay, 1995, p. 9.)

Leur étonnante régularité au sein du discours public entourant l'exposition me laisse penser qu'outre cet impérialisme de la trace des sociétés occidentales contemporaines qu'évoque Nora, de tels énoncés concourent à la production de la mémoire musicale en tant que responsabilité civique individuelle. Certes, le Musée paraît se substituer aux artistes en tant que gardien institutionnel autorisé des souvenirs, conservateur au moins temporaire des richesses cachées d'un patrimoine musical dont les sujets acteurs ignoreraient l'existence ou sous-estimeraient l'importance. Mais par le biais d'objets fétiches extraits de l'environnement domestique d'artistes qui sont aussi, comme tout autre citoyen, des individus propriétaires ou des locataires, célibataires ou mariés, l'exposition a aussi pour effet de faire de chaque Québécois ou Québécoise le détenteur ou la détentrice potentielle d'une parcelle de cette mémoire musicale collective dont le souvenir serait moins un privilège qu'une responsabilité, sinon une obligation. Collectionner ne consisterait pas en un simple passe-temps réservé aux admirateurs ou admiratrices fanatiques, aux spécialistes d'un genre donné, aux individus fortunés ou aux agents professionnels des institutions dont c'est le mandat ; cela devient en quelque sorte un réflexe à développer, un effort à faire pour tous ceux et celles qui veulent se souvenir et contribuer à l'instauration d'une mémoire musicale « nationale ». En proposant ainsi la mémoire en partage, le récit de la réussite que raconte le musée fait en sorte que la musique populaire devienne quelque chose d'important pour tous les citoyens culturels du Québec.

\section{DIS-MOI CE DONT TU TE SOUVIENS, JE TE DIRAI QUI TU ES...}

L'exposition « Je vous entends chanter » propose en quelque sorte un voyage au cœur d'une québécitude dont la musique populaire, plus particulièrement la chanson, constituerait tant l'élément moteur que le produit et le miroir. Tout en évitant le piège d'un nationalisme étroit et nombriliste, l'exposition participe à l'articulation de l'identitaire québécois en tant que projet de « gouvernement des conduites » (Foucault, 1994) axé exclusivement sur la nation. Médiatisée par les passés « présents » des expériences individuelles et collectives qu'il convoque, la production de cet identitaire constitue un processus d'identification continuelle à ce que la musique populaire révèle de ce que « nous » avons été et de ce que « nous » sommes déjà : un renvoi perpétuel au " peuple québécois » en tant que référence déjà constituée qu'autorise et légitime à la fois cette mise en musée de la chanson québécoise, voire du Québec en musique. Le dispositif dont cet événement muséal est tributaire opère de façon à lier de diverses façons la trajectoire collective du « peuple québécois » aux différents parcours des individus ou groupes producteurs et consommateurs qu'incarnent les sous-ensembles contrastés de produits, d'artistes et d'objets associés à la chanson. L'exposition concourt ainsi à la production discursive d'une citoyenneté culturelle (Allor et Gagnon, 1994) en vertu de laquelle le statut de Québécois ou Québécoise ne s'acquiert pas simplement par quelque contrat légal qui, liant le sujet à l'État dont il ou elle constitue l'un des membres souverains, détermine leurs droits et responsabilités respectifs; il procède de la reconnaissance et de l'expérience sans cesse renouvelées d'une 
culture, en l'occurrence musicale, québécoise intégrée et intégratrice, dont le sujet et la nation à laquelle il ou elle est conduit à s'identifier sont de quelque manière solidaires. Les « Je me souviens » que la visite de « Je vous entends chanter » a pu susciter comptent au nombre des traces d'un processus de mise en société qui fait de la musique populaire le lieu d'articulation privilégié du passé comme du devenir du Québec et de ses citoyens.

\title{
REMERCIEMENTS
}

La recherche sur laquelle repose cet article n'aurait pas été possible sans la généreuse et amicale collaboration du personnel du Musée de la civilisation de Québec; je tiens à remercier tout particulièrement Dominique Bilodeau, Marie-Josée Des Rivières, Louise Goupil et Guylaine Blouin pour leur aide inestimable. Merci aussi à Elspeth Probyn, Martin Allor, Tony Bennett, Roger de la Garde et Val Morrison pour leurs commentaires sur une première version de cet article. J'aimerais aussi remercier le Conseil de recherches en sciences humaines du Canada et le Fonds FCAR pour leur appui financier.

\author{
Line GRENIER \\ Département de communication \\ Université de Montréal \\ C.P. 6128, Succ. Centre-ville \\ Montréal (Québec), Canada \\ $\mathrm{H} 3 \mathrm{C} 3 \mathrm{~J} 7$
}

\section{RÉSUMÉ}

L'auteure s'intéresse à la première exposition d'envergure nationale consacrée à la musique populaire québécoise, « Je vous entends chanter », en tant que terrain clé pour l'étude du dispositif qui rend possible l'articulation de ce domaine musical comme lieu privilégié de production de la citoyenneté culturelle et de l'identitaire au Québec. Les documents et artefacts composant l'exposition, ainsi que la couverture médiatique dont elle a fait l'objet, servent ici à l'examen des conditions matérielles, institutionnelles et discursives, qui concourent à faire de l'histoire de la musique populaire celle du «peuple québécois ». L'analyse explore le jeu compiexe des mémoires qui médiatisent les façons contrastées dont cet événement muséal dit, nomme et raconte la place de la musique populaire québécoise et, ce faisant, les formes d'allégeance, d'identification et d'appartenance qui y sont articulées.

\section{SUMMARY}

The author has examined the first exhibition of national scope dedicated to Quebec popular music, Je vous entend chanter, as a key area for the study of the mechanism by which this type of music functions as a pivotal point in the production of facets of citizenship related to culture and identity. The documents and artifacts making up the exhibition, as well as the media coverage it received, serve to examine the material, institutional and discursive conditions which come together to make the history of popular music the history of the "Quebec people". The analysis explores the complex interplay of memories that promote the contrasting ways in which the event states, names and relates the place of popular Quebec music, and, in so doing, the forms of allegiance, identification and belonging which are linked to it.

\section{RESUMEN}

La autora se interesa en la primera exposición de envergadura nacional consagrada a la música popular quebequense, $J_{e}$ vous entends chanter, como un terreno clave para el estudio del dispositivo que hace posible la articulación de ese dominio musical como lugar privilegiado de producción de la ciudadanía cultural y de la identidad en Québec. Los documentos y artefactos que componen la exposición, así como la cobertura mediática de la que ella fue objeto, sirven aquí al examen de las condiciones materiales, institucionales y discursivas, que concurren a hacer la historia de la música popular, la del « pueblo quebequense ». El análisis explora el juego complejo de las memorias que mediatizan las maneras contrastadas en las cuales este evento museológico dice, nombra y cuenta el lugar de la música popular quebequense y, al hacerlo, las formas de fidelidad, de identificación y de pertenencia que sont allí articuladas.

\section{BIBLIOGRAPHIE}

ALLOR, Martin (1997), «Locating Cultural Activity: the « Main " as Chronotope and Heterotopia ", Topia (1) 1, pp. $42-54$. 
ALLOR, Martin et Michelle GAGNON (1994), L'État de culture. Généalogie discursive des politiques culturelles québécoises, Montréal, Groupe de recherche sur la citoyenneté culturelle (GRECC), Université Concordia et Université de Montréal.

ANDERSON, Benedict (1991), Imagined Communities, Londres, Verso.

ARPIN, Koland (1992), Le Musée de la civilisation. Concepts et pratiques, Québec, Éditions MultiMondes et Musée de la civilisation.

BAILLAKGEON, Richard et Christian CÔTÉ (1991), Destination Ragou. Une histoire de la musique populaire au Québec, Montréal, Triptyque.

BILODEAU, Dominique (1994), La, La, La Chanson. Concept (projet 364), Québec, Musée de la civilisation, Service des expositions.

CAISSE et CHARTIER (1995a), transcription du texte de l'émission «Inside Québec » CKMI-TV, 11 novembre, Montréal, Le réseau national d'extraits de presse du Québec et du Canada.

CAISSE et CHARTIER (1995c), transcription du texte de l'émission «CBV-Matin », CBVQ, 18 octobre, Montréal, Le: réseau national d'extraits de presse du Québec et du Canada.

CAISSE at CHARTIER (1996), transcription d'une émission diffusée sur les ondes de Radio-Canada International/ Qıébec, Jean-François Bolduc journaliste, 23 février, Montréal, Le réseau national d’extraits de presse du Québec et du Canada.

CHAMBIERLAND, Roger et André GAULIN (1994), La chanson québécoise. De la Bolduc à aujourd'hui. Anthologie, Montréal, Nuit Blanche.

DE CERTEAU, Michel (1990), L'invention du quotidien. I-Arts de faire, Paris, Folio, Essais.

FOUCAULT, Michel (1969), L'archéologie du savoir, Paris, Seuil.

FOUCAULT, Michel (1980), «Power and Strategies », in C. Gordon (ed.), Power/Knowledge : Selected Interviews and Oiker Writings 1972-1977, New York : Pantheon.

FOUCAULT, Michel (1994), «Entretien avec Michel Foucault », 1977, D. Defert et F. Erals (sous la dir.), Dits et écrits, vol. 1, Paris, Gallimard, pp. 140-160.

FRITH, Simon (1987), «Towards an Aesthetic of Popular Music », in R. Leppert et Susan McClary (éds.), Music and Society. The Politics of Composition, Perfomance and Reception, Cambridge, Cambridge University Press, pp. 133-149.

GIROUX, Robert et al. (1991), Le guide de la chanson québécoise, Montréal, Triptyque.

GRANGER, Gilles-Gaston (1988), Essai d'une philosophie du style [1968], Paris, Editions Odile Jacob.

GRENIER, Line, (1993), "The Aftermath of a Crisis : Québec Music Industries in the 1980s ", Popular Music, vol. 11, $\mathrm{n}^{\circ} 2$, pp. 221-233.

GRENIER, Line (1994), « Policing French-Language Music on Canadian Radio : The Twilight of the Popular Record Era ? » in Tony Bennett, Simon Frith, Larry Grossberg, John Shepherd et Graeme Turner (éds.), Rock and Popular Music : Politics, Policies and Institutions, Londres, Routledge, pp. 203-219.

GRENIER, Line, Claude MARTIN et Fabien DÉGLISE (1996), « Processes of Valorization in Québécois Popular Music ", conférence prononcée dans le cadre du colloque annuel de l'Association canadienne de communication, Sainte-Catherine (Ontario), juin.

GROSSEERG, Lawrence (1992), We Gotta Get Out Of This Place. Popular Conservatism and Postmodern Culture, Londres, Routledge.

HALL, Stuart (1986), « On Postmodernism and Articulation : an Interview with Stuart Hall » Joumal of Communication Inquin, 10 (2), p. 45-60.

HAVERCROFT, Barbara (1993), «Énonciation/Énoncé », in Irena R. Makaryk (éd.), Encyclopedia of Contemporary Literary Theon, Toronto, University of Toronto Press, p. 540-543.

HENNION, Antoine (1993), La passion musicale, Paris, Métaillé.

LE MENESTREL, Sara (1996), « La collecte de l'objet contemporain. Un défi posé au Musée de la Civilisation à Québec ", Ethnologie française, XXXVI, p. 74-91.

LEPAGE, Jocelyne (1995), «Une histoire d'amour entre un peuple et sa chanson », La Presse, Arts et Spectacles, 4 novembre, p. D-19.

MASSEY, Doreen (1993), « Power Geometry and a Progressive Sense of Place ", in J. Bird et al., Mapping the Futures: Local Cultures, Global Changes, London, Routledge.

MUSÉE DE LA CIVILISATION (1995), I Hear You Singing, Je vous entends chanter. Guidebook, Service des expositions.

MUSÉE DE LA CIVILISATION (1996), Je vous entends chanter. Recueil des textes de l'exposition, Québec, Service des expositions.

NORA, Jierre (éd.) (1984), Les lieux de mémoire, Paris, Gallimard.

RÉCANATI, François (1979), La transparence et l'énonciation, Paris, Seuil.

RIOUX, Lucien (1992), 50 ans de chanson française. De Trenet à Bruel, Paris, LAArchipel.

SAINT-JACQUES, Denis et al. (1995), Ces livres que vous avez aimés, Montréal, Nuit Blanche.

SLACK, Jennifer Daryl (1996), "The Theory and Method of Articulation », Stuart Hall Critical Dialogues in Cultural Sirudies, D. Morley et K.-H. Chen éditeurs, Londres, Routledge.

TODOROV, Tzvetan (1995), Les abus de la mémoire, Bruxelles, Arléa.

TREMBLAY, Régis (1995), "Le Québec profond parle en chanson », Le Soleil, 18 oct., p. B-4.

WHITE, Hayden (1987), The Content of the Form. Narrative Discourse and Historical Representation, Baltimore, The John Hopkins University Press. 